\title{
Confirmed Response Minimum Duration
}

National Cancer Institute

\section{Source}

National Cancer Institute. Confirmed Response Minimum Duration. NCI Thesaurus. Code C98715.

The minimum amount of time required to meet the criteria for a confirmed response to treatment. 